\title{
The Curious Quantum Mechanics of Pre- and Post-Selected Ensembles
}

\author{
Wayne $\mathrm{Hu}^{1}$ \\ Received July 28, 1989
}

Despite claims to the contrary, the curious statistical properties of pre- and postselected ensembles are neither impossible nor surprising. The properties of weak measurements on such ensembles are examined and shown to be in complete accord with ordinary quantum mechanics.

\section{INTRODUCTION TO PRE- AND POST-SELECTED ENSEMBLES}

In a provocative paper, ${ }^{(1)}$ Albert, Aharonov, and D'Amato proposed an analysis of pre- and post-selected ensembles in quantum mechanics (PPS ensembles) which seemed to show that in the interval between pre- and post-selection, certain noncommuting observables all have determinate values. Moreover, these values are context dependent: The value assumed by a degenerate observable (e.g., an observable represented by a onedimensional projection operator) depends on what other observables are selected in a measurement of a complete commuting set with $P$ (i.e., the value of the one-dimensional projection operator, 1 or 0 , depends on the choice of a complete commuting set of basis vectors spanning the Hilbert space).

The fallacy in this argument was pointed out by Bub and Brown. ${ }^{(2)}$ They showed that the identity of a PPS ensemble depends on the measurement(s) made on the system in the ensemble in the interval between the pre- and post-selection. For the example proposed by Albert, Aharonov, and D'Amato, Bub and Brown demonstrated that the PPS ensembles are manifestly different. They went on to construct an example

\footnotetext{
${ }^{1}$ Department of Physics, Jadwin Hall, Princeton University, Princeton, New Jersey 08544.
} 
in which the PPS ensembles are not manifestly different and exhibit a peculiar contextuality as well as nonlocality for the values assumed by certain noncommuting observables in the interval between pre- and postselection.

However, although the ensembles are not manifestly different, there is no reason to believe that the ensembles are identical, as Bub and Brown argued. Indeed, we have every reason to believe otherwise, on the basis that any precise measurement of an observable $P$ will disturb other observables that do not commute with $P$. For an individual quantum system in a PPS ensemble (pre-selected for a particular value of an observable $B$ ), it is not the case in general that the system would have been post-selected after an intervening measurement of an observable $Q$ if the system was in fact post-selected after an intervening measurement of an observable $P$ not commuting with $Q$.

Vaidman, Aharonov, and Albert ${ }^{(3)}$ exploit the case considered by Bub and Brown to show that for certain PPS ensembles one can infer the results of an intervening measurement on any one of the three spin components $\sigma_{x}, \sigma_{y}, \sigma_{z}$. It is not apparent why this result should have any more intrinsic interest than the Bub and Brown example. Again, the inference from the statistics of the PPS ensemble to precise values for $\sigma_{x}, \sigma_{y}$, and $\sigma_{z}$ is allowed in standard quantum theory since the identity of the PPS ensemble depends on whether $\sigma_{x}, \sigma_{y}$, or $\sigma_{z}$ was in fact measured in the interval between the pre- and post-selection.

Aharonov, Albert, et al. apparently accept this fundamental criticism of PPS ensembles: the ensemble is not uniquely defined by the pre- and post-selection states. The identity of the PPS ensemble depends upon the measurement taken in the intervening period. One can neither infer retrodictively the determinateness of noncommuting observables nor show that probability assignments for measurements depend on context. The problem is that any such intervening measurement will fundamentally disturb the system measured and so alter the PPS ensemble. Perhaps these criticisms drove the authors to examine the possibility of nondisruptive intervening measurements more closely. In a series of papers beginning in 1987, Aharonov, Albert, et al. ${ }^{(4,5)}$ analyze the results of taking such weak or imprecise measurements on PPS ensembles. They conclude that one obtains surprising results, some of which seem classical as opposed to quantum mechanical, some of which seem altogether impossible. They believe that they have discovered a new quantum variable, the so-called weak value that governs the PPS ensemble. They claim that such ensembles in which an imprecise measurement has taken place generate "curious" and "paradoxical" results. The purpose of this paper is to examine the validity of these claims for weak measurements. 


\section{WEAK QUANTUM MEASUREMENTS ON PPS ENSEMBLES}

\subsection{Realistic Measurements: The Standard Prescription}

The authors ask us to consider the standard method of obtaining a precise measurement on a system. Say we wish to measure the $X$ observable of a system. We have furthermore at our disposal a measuring device containing the canonical variable $Q$. The measurement Hamiltonian is given by a normalized function $g(t)$ which couples the observable and the measurement variable:

$$
H=-g(t) Q X
$$

If, as in this case, the Hamiltonian commutes with its time derivative, we may write the unitary time translation operator as

$$
U(t)=\exp \left[-i \int H(t)\right]
$$

Now consider the realistic case in which the initial state of the measuring device is a Gaussian centered around zero:

$$
\psi_{i}=\frac{1}{\sqrt{\Delta}(2 \pi)^{1 / 4}} \exp \left(-q^{2} / 4 \Delta^{2}\right)
$$

or in terms of the canonical momentum $\pi$ where $[q, \pi]=i$

$$
\phi_{i}=\frac{1}{\sqrt{\Delta \pi}(2 \pi)^{1 / 4}} \exp \left(-\pi^{2} / 4(\Delta \pi)^{2}\right), \quad \Delta \pi=1 / 2 \Delta
$$

Let the initial state of the system be the superposition:

$$
\chi_{i}=\sum_{i} \alpha_{n}\left|x_{n}\right\rangle
$$

In order to find the state of the measuring device after the interaction $\phi(t)$, we operate on the composite system with the unitary time translation operator:

$$
\phi(t) \chi(t)=U(t) \phi_{i} \chi_{i}
$$

In a representation where the Hamiltonian is diagonal, the operation yields

$$
\phi(t) \chi(t)=\exp \left[-i \int H d t\right] \frac{1}{\sqrt{2 \pi}} \int \psi_{i} \exp (-i \pi q) d q \sum_{n} \alpha_{n}\left|x_{n}\right\rangle
$$


Thus,

$$
\begin{array}{r}
\phi(t) \chi(t)=\sum_{n} \alpha_{n} \frac{1}{\sqrt{2 \pi}} \int \psi_{i} \exp \left(-i \pi\left(q-x_{n}\right) d q\left|x_{n}\right\rangle\right. \\
\phi(t) \chi(t) \propto \sum_{n} \alpha_{n} \exp \left[-\left(\pi-x_{n}\right)^{2} / 4(\Delta \pi)^{2}\right]\left|x_{n}\right\rangle
\end{array}
$$

The measuring device is left in a state which is a superposition of Gaussians, shifted by the eigenvalues of $X$, with the weights of the original $X$ distribution. This macroscopic state of the measuring device can then be observed, collapsing the superposition onto a sharp value for the canonical variable $\pi$. If $\Delta \pi \ll \Delta x_{n}$, i.e., the width of each Gaussian is sufficiently small, one can determine an unambiguous value for $X$ since there is little overlap to confuse the measurement. This is, for example, how a Stern-Gerlach magnet works. Consider a spin-1/2 particle traveling in the $\mathbf{y}$ direction with momentum $p_{0}$. At $y_{1}$, position a magnetic field gradient in the $\mathbf{z}$ direction. As in the standard prescription, the spin of the particle interacts with the canonical position variable during its passage through the Stern-Gerlach magnet:

$$
\begin{aligned}
H & =-g\left(y-y_{1}\right) \mu\left(\partial B_{z} / \partial z\right) \sigma_{z} z \\
& =-g\left[p_{0} / m\left(t-t_{1}\right)\right] \chi_{i} q_{i}
\end{aligned}
$$

The result is a change in the momentum in the $z$ direction which causes an easily detectable position separation. Note that such a measurement does not collapse the wavepacket; the result remains a pure state rather than a mixture. With the proper field gradient, we may reverse the separation to obtain the original wavefunction. This fact will become important as we investigate the interference properties of such a system.

Aharonov, Albert, et al. ask us to consider the opposite limit: an imprecise measurement where $\Delta \pi \gg \Delta x_{n}$. Here we are "measuring" the difference in shifts of the Gaussian curve which are much smaller than the width of the original Gaussian. They note that though a single measurement cannot yield any information due to the large uncertainty in the measuring device, measurements on a large ensemble of $N$ particles similarly prepared can reduce the statistical error by $N^{-1 / 2}$. A sufficiently large ensemble will produce an arbitrarily accurate determination of the average $\langle X\rangle$ for the ensemble. They proceed to analyze the expected results of such measurements of PPS ensembles.

\subsection{The Curious Statistics of Weak Measurements of PPS Ensembles}

The authors show that such measurements yield results that seem impossible and defy quantum mechanical common sense. They refer to 
such results as "impossible" and "something of a miracle." ${ }^{(4)}$ Consider a pre-selection process that places the system to be measured in an initial state $\chi_{i}$. The composite system, as before, is given by

$$
\exp \left(-i \int H d t\right) \chi_{i} \phi_{i}
$$

where $H(t)$ is the time-dependent Hamiltonian of the intermediate weak measurement. Now post-select for $\chi_{f}$ by taking the projection of the timedependent $\chi(t)$ onto $\chi_{f}$

$$
\left|\chi_{f}\right\rangle\left\langle\chi_{f}\left|\exp \left(-i \int H d t\right)\right| \chi_{i}\right\rangle \phi_{i}
$$

For weak coupling Aharonov, Albert, et al. derive

$$
\begin{aligned}
\left\langle\chi_{f}\left|\exp \left(-i \int H d t\right)\right| \chi_{i}\right\rangle & =\sum_{n} \frac{(i q)^{n}}{n !}\left\langle\chi_{f}\left|X^{n}\right| \chi_{i}\right\rangle \\
& \approx\left\langle\chi_{f} \mid \chi_{i}\right\rangle\left(1+\frac{i q\left\langle\chi_{f}|X| \chi_{i}\right\rangle}{\left\langle\chi_{f} \mid \chi_{i}\right\rangle}\right) \\
& \approx\left\langle\chi_{f} \mid \chi_{i}\right\rangle \exp \left(\frac{i q\left\langle\chi_{f}|X| \chi_{i}\right\rangle}{\left\langle\chi_{f} \mid \chi_{i}\right\rangle}\right)
\end{aligned}
$$

which tells us that the shift in momentum is given by

$$
X_{w}=\frac{\left\langle\chi_{f}|X| \chi_{i}\right\rangle}{\left\langle\chi_{f} \mid \chi_{i}\right\rangle}
$$

which they call the weak quantum value for the measured observable. Under the traditional prescription for precise measurements, this value would represent the result of a measurement of the $X$ observable. However, note that we have obtained something rather disturbing. The quantity $X_{w}$ is not bounded by the possible eigenvalues of $X$. The inner product of the initial and final states in the denominator can become vanishingly small. For example, if the process represented a Stern-Gerlach measurement of spin for a spin-1/2 system, Aharonov et al. ${ }^{(5)}$ noted that we might get a result of 100 . What is happening here? It would seem that the mathematical derivation of Aharonov, Albert, et al. obscures the actual physics of the process. Let us reconsider the experiment from the perspective of ordinary quantum mechanics. For definiteness, let us employ a Stern-Gerlach measurement on a spin-1/2 particle as in the case considered by the authors. Pre-select a beam of spin-1/2 particles moving in the $\mathbf{y}$-direction 
for spin up in the $\xi$ direction lying in the plane spanned by $\mathbf{x}-\mathbf{z}$ and separated by an angle $\alpha$ from $\mathbf{x}$. Let the weak measurement be on $\sigma_{z}$ and the post-selection be for $\sigma_{x}$ up. For convenience, let us define an additional angle $\beta=\alpha-\pi / 2$ as that which separates the $\xi$ and $\mathbf{z}$ axes (see Fig. 1). The initial spin state in the $z$-representation is

$$
\chi_{i}=\cos \beta / 2\left|z_{+}\right\rangle+\sin \beta / 2\left|z_{-}\right\rangle
$$

As above, after the measurement the overall system of measuring device and particle spin is left in the following superposition of states:

$$
\begin{gathered}
\left.\phi_{w} \chi_{w} \propto\left(\cos \beta / 2 z_{+}\right\rangle\right) \exp \left[-(\pi-\delta \pi)^{2} / 4(\Delta \pi)^{2}\right] \\
+\left(\sin \beta / 2\left|z_{-}\right\rangle\right) \exp \left[-(\pi+\delta \pi)^{2} / 4(\Delta \pi)^{2}\right] \\
\delta \pi=\mu\left(\partial B_{z} / \partial z\right)
\end{gathered}
$$

If we now post-select only for $\sigma_{x}$ up, we obtain the final state

$$
\begin{aligned}
\phi_{f} \chi_{f}= & \left|x_{+}\right\rangle\left(-\cos \beta / 2 \exp \left[-(\pi-\delta \pi)^{2} / 4(\Delta \pi)^{2}\right]\right. \\
& \left.+\sin \beta / 2 \exp \left[-(\pi+\delta \pi)^{2} / 4(\Delta \pi)^{2}\right]\right)
\end{aligned}
$$

via the identity

$$
\left|z_{+}\right\rangle=\frac{1}{\sqrt{2}}\left[-\left|x_{+}\right\rangle+\left|x_{-}\right\rangle\right]\left|z_{-}\right\rangle=\frac{1}{\sqrt{2}}\left[\left|x_{+}\right\rangle+\left|x_{-}\right\rangle\right]
$$

Our weak measurement of $\sigma_{z}$ separated the initial state spatial wave packet into a superposition of two slightly shifted Gaussian distributions corresponding to the different spin states. It is important to note that our intermediate state is a superposition rather than a mixture. The weak measurement in effect created a $\pi / 2$ phase shift for the post-selected $\sigma_{x}$ up wave functions. The final state is therefore a superposition of two slightly dis-

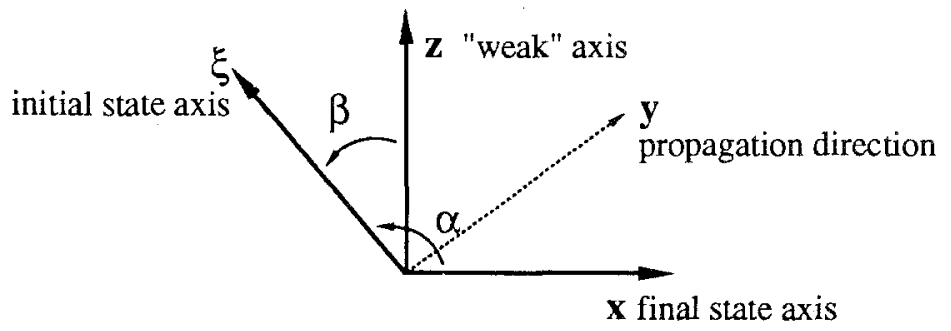

Fig. 1. Weak Stern-Gerlach measurement. 
placed Gaussian wavepackets $\pi / 2$ out of phase. It is no surprise that we obtain destructive interference resulting in a measured state with a large component of momentum in the $\mathrm{z}$ direction. Since the value for the shift in momentum is taken as the measurement of spin, one is tempted to state that an unexpectedly large value for spin has been measured. Such an inference is not warranted. The cause of the effect lies in the measurement prescription rather than the spin of the particle.

Aharonov, Albert, et al. give the dependence of this counterintuitively large shift as a function of separation angle $\alpha$ (or $\beta$ ). We can easily derive the result from the general prescription for the weak quantum value given above:

$$
\begin{aligned}
\delta p_{z}= & \mu\left(\partial B_{z} / \partial z\right) \frac{\left\langle x_{+}\left|\sigma_{z}\right| \xi_{+}\right\rangle}{\left\langle x_{+} \mid \xi_{+}\right\rangle} \\
\left\langle x_{+}\left|\sigma_{z}\right| \xi_{+}\right\rangle= & \left(\frac{1}{\sqrt{2}}\left\{-\left\langle z_{+}\right|+\left\langle z_{-}\right|\right\}\right) \\
& \times \sigma_{z}\left(\frac { 1 } { \sqrt { 2 } } \left\{[\cos \alpha / 2+\sin \alpha / 2]\left|z_{+}\right\rangle\right.\right. \\
& \left.\left.+[\sin \alpha / 2-\cos \alpha / 2]\left|z_{-}\right\rangle\right\}\right) \\
\delta p= & \mu\left(\partial B_{z} / \partial z\right) \frac{\sin \alpha / 2}{\cos \alpha / 2} \\
\delta p= & \mu\left(\partial B_{z} / \partial z\right) \tan \alpha / 2
\end{aligned}
$$

Thus the weak value for spin is given by $\sigma_{w}=\tan \alpha / 2$. Let us see how our derivation compares with this result. First note that the general form is correct:

$$
\phi_{f} \chi_{f} \propto \exp \left[-(\pi-\delta \pi)^{2} / 4(\Delta \pi)^{2}\right]-\tan \beta / 2 \exp \left[-(\pi+\delta \pi)^{2} / 4(\Delta \pi)^{2}\right]
$$

Take the extreme case where $\beta=\pi / 2$. As $\delta \pi \rightarrow 0$ we have complete cancellation. For a $\beta<\pi / 2$ and finite $\delta \pi$, we have near cancellation in the central region with only a fringe distribution at $\pi \geqslant \delta \pi$. This corresponds to the shift to infinity at $\alpha=\pi$. Now let us take a careful look at where the peak of our superposition lies:

$$
\begin{aligned}
\partial \phi_{f} \chi_{f} / \partial \pi \propto & (\pi-\delta \pi) \exp \left[-(\pi-\delta \pi)^{2} / 4(\Delta \pi)^{2}\right] \\
& -(\pi+\delta \pi) \tan \beta / 2 \exp \left[-(\pi+\delta \pi)^{2} / 4(\Delta \pi)^{2}\right]
\end{aligned}
$$


We expect that the shift in the Gaussians will be relatively unimportant in comparison to $\delta \pi$ :

$$
\begin{aligned}
\left(\pi_{\max }-\delta \pi\right) & =\left(\pi_{\max }+\delta \pi\right) \tan \beta / 2 \\
\pi_{\max } & =\frac{1+\tan \beta / 2}{1-\tan \beta / 2} \delta \pi \\
\pi_{\max } & =\tan (\beta / 2+\pi / 4) \delta \pi \\
\pi_{\max } & =\tan \alpha / 2 \delta \pi \\
\pi_{\max } & =\mu d B / d z \tan \alpha / 2
\end{aligned}
$$

So we obtain the same result as the authors using a standard quantum mechanical argument. Indeed, our puzzlement has vanished. The anomalously large shift arises from a completely logical and predictable destructive interference effect. The prose and terse mathematical derivation of the authors obscures this fact. This effect is no more disturbing than a double-slit interference pattern.

\subsection{The Physical and Foundational Significance of Weak Measurements}

Aside from exclamations of wonderment, the authors are rather vague as to the significance of their result. In their 1987 paper, ${ }^{(4)}$ they make several general claims which they leave undeveloped. They state that weak measurements have a commutative and classical nature. It does not seem surprising that by sacrificing precision in employing a "fuzzy" or classical type measurement that we re-obtain some degree of "commutivity," However, if we view measurement disturbance as the fundamental problem with the originally presented interpretation of PPS ensemble statistics, we might understand the possible importance of this recovery for the interpretation of PPS ensembles.

Recall that we have shown that the PPS ensemble is not uniquely specified by the pre- and post-selection states. The very identity of the subensemble depends on what measurements were taken in the intervening period. The disturbance caused by the measurements of noncommuting variables forbids us to interpret PPS inferences as possessing reality in the EPR sense. It is not the case that we are presented with a system in which we may measure one of either noncommuting variable and obtain a certain result with probability unity. Our choice of measurements changes the system under consideration. Even if the overall statistics for the subensemble are the same, it does not follow that we can extend the argument to include specific systems. A particular system which is pre- and postselected under one intervening measurement may not necessarily be under 
another. All these caveats arise because the intervening measurement disturbs the other observables of the system in question. If, however, the measurements are significantly weakened to the point at which they cause no fundamental disturbance, we might be tempted to assert that the identity of the PPS ensemble is independent of any weak measurements taken in the intervening period. Aharonov, Albert, et. al. ${ }^{(4)}$ ask us to reconsider the experiment in which we pre-select for $\sigma_{x}$ up.and post-select for $\sigma_{y}$ up. Recall that any precise measurement of $\sigma_{x}$ in the intervening period will yield spin up with probability unity; any precise measurement of $\sigma_{y}$ will also yield spin up with probability unity. However, it does not follow that both values are simultaneously definite. We can easily see this by the fact that if in the intervening period we measured both $\sigma_{y}$ and $\sigma_{x}$ in that order we can no longer predict either to probability unity. The disturbance of one upon the other opens up other possible paths to the post-selected state.

The authors claim that one may avoid this problem by sufficiently weakening the measurement. They seem to be making the following argument: A weak measurement on $\sigma_{y}$ disturbs each individual system insignificantly so that the final state selection remains unchanged for the case in which it is made and in which it is not. The authors have in mind a weak measurement in the sense of a Stern Gerlach separation of the initial wave packet into a superposition of spatially displaced components. We can "verify" the result of an intermediate measurement by observing where the peak in the final state Gaussian lies. We expect that the location is shifted by a small amount corresponding to the result of the weak measurement as spin up. By observing a large number of similarly prepared systems we can measure the expectation value of the weak measurement as up, to arbitrary accuracy. Of course, if we now replace the weak measurement of $\sigma_{y}$ by one of $\sigma_{x}$ we have also changed nothing since the initial state is an eigenstate of $\sigma_{x}$. The same logic follows for a measurement of $\sigma_{x}$ followed by $\sigma_{y}$. If such an argument for a weak measurement $\sigma_{y}$ followed by one of $\sigma_{x}$ holds true, as the authors claim, we might be justified in asserting that both weak variables are simultaneously definite during the intervening period between selection measurements. We need, however, to analyze this assertion more closely.

Let us consider the measurements involved: $\sigma_{y}$ followed by $\sigma_{x}$. The initial state is spin up in the $\mathbf{x}$ direction:

$$
\left|\chi_{i}\right\rangle=\left|x_{+}\right\rangle
$$

A weak measurement of $\sigma_{y}$ splits the wavefunction into a superposition:

$$
\frac{1}{\sqrt{2}}\left[\left|y_{+}\right\rangle(\delta y)+\left|y_{-}\right\rangle(-\delta y)\right]
$$


where $\delta y$ represents an infinitesimal shift in the $p_{y}$ Gaussian distribution. The authors assert that if at this time we collapsed the wave packet for those systems which would have been destined to contribute to the PPS ensemble, only the up component would be found. Let us, however, follow the measurement through. A weak measurement of $\sigma_{x}$ further splits the wave packet into:

$$
\begin{aligned}
\frac{1}{2}\left[\mid x_{+}\right. & >(\delta y)(\delta x)-\left|x_{-}\right\rangle(\delta y)(-\delta x) \\
& \left.+\left|x_{+}\right\rangle(-\delta y)(\delta x)+\left|x_{-}\right\rangle(-\delta y)(-\delta x)\right]
\end{aligned}
$$

The post selection for $\sigma_{y}$ up projects out the spatial wave function:

$$
\begin{aligned}
& \approx \frac{1}{2}[(\Delta y+\delta y)(\delta x)+(\Delta y+\delta y)(-\delta x)+(\Delta y-\delta y)(\delta x)-(\Delta y-\delta y)(-\delta x)] \\
& \approx(\Delta y)
\end{aligned}
$$

where the $\Delta y$ 's represent an unambiguously shifted Gaussian created by the precise measurement.

In what way can we say the weak measurements commute? We see that after the weak $\sigma_{x}$ measurement we obtain a state where the down components very nearly cancel due to the extremely small difference between $\delta x$ and $-\delta x$. Despite the intervening $\sigma_{y}$ measurement, we obtain essentially spin up in the $x$ direction. The quantity $\sigma_{x}$ effectively does not "see" $\sigma_{y}$. However, let us examine what happens to the down component "result" of the weak measurement $\sigma_{y}$. This component follows through all the way to the final state. It is not the case that for all systems which yield $\sigma_{y}$ up in the final state, $\sigma_{y}$ was up during the weak measurement (in the above sense). We can see this in the fact that if we now collapse the final state Gaussian, the expectation value would by at $\Delta y$ rather than $\Delta y+\delta y$ as the authors assert. It remains that the ensemble is fundamentally disturbed by the extra intervening measurement of weak $\sigma_{x}$ which, like the precise measurement, opened up other possible paths to the final state. On the level of individual particles, the resulting PPS ensembles are different. Arguments for even simultaneous weak values of noncommuting observables for the intervening period between pre- and post-selection as well as contextuality are still blocked.

The authors further believe that the supposed commutivity and the curious statistics of the weak measurements indicate that the elements in the system "conspire together to point to an internally consistent picture of a classical rather than a quantum-mechanical system." ${ }^{(4)}$ In particular, they have in mind that values for angular momentum components add vectorially to assign values to spin in all directions. Under the hypothesis that $\sigma_{x}$ and $\sigma_{y}$ have determinate weak values in the intervening period, which 
as we have seen is misguided, they propose a thought experiment. Imagine that we reduced the time between the two to zero. Essentially then, we are measuring spin in a new direction which is a linear combination of $\sigma_{x}$ and $\sigma_{y}$. The authors defend this picture by showing that for the case of a measurement of spin at a $\pi / 4$ angle from $x$ and $y$, the statistics generated by the weak measuring prescription are exactly those of a classical addition of two unit spin vectors: one in the $\mathbf{x}$ and one in the $\mathbf{y}$.

Let us show a stronger version of this argument that would lead one to believe that this type of classical description is indeed a valid account of the process. Consider spin along an axis in the direction $\alpha$ which makes an angle $\theta$ with $\mathbf{x}$. Classically, if for the considered ensemble $\sigma_{x}=\sigma_{y}=1$ at the time of the weak measurement, we would expect that the magnitude of $\sigma_{\alpha}$ is just given by the projection of these two unit vectors onto the $\alpha$ direction:

$$
\sigma_{\alpha}=\cos \theta+\sin \theta
$$

The prescription for weak measurements gives the weak spin as

$$
\begin{aligned}
\sigma_{w_{\alpha}} & =\frac{\left\langle y_{+}\left|\sigma_{\alpha}\right| x_{+}\right\rangle}{\left\langle y_{+} \mid x_{+}\right\rangle} \\
\sigma_{\alpha}\left|x_{+}\right\rangle & =\cos \theta / 2\left|\alpha_{+}\right\rangle-\sin \theta / 2\left|\alpha_{-}\right\rangle \\
\left|y_{+}\right\rangle & =\frac{-1}{\sqrt{2}}[\cos \theta / 2+\sin \theta / 2]\left|\alpha_{+}\right\rangle+\frac{1}{\sqrt{2}}[\cos \theta / 2-\sin \theta / 2]\left|\alpha_{-}\right\rangle \\
\sigma_{w_{\alpha}} & =\cos ^{2} \theta / 2-\sin ^{2} \theta / 2+2 \cos \theta / 2 \sin \theta / 2 \\
& =\cos \theta+\sin \theta
\end{aligned}
$$

It would seem then that the classical description is correct: the $\sigma_{x}$ and $\sigma_{y}$ angular momentum vectors are simply added vectorially. Could it be that we have discovered one way in which realistically weak measurements yield classical results? No. We can easily see that this case is just a matter of coincidence by returning to the case considered above in which the initial and final spin-up vectors were separated by an angle greater than $\pi / 2$. Here we obtained from the weak prescription $\sigma_{\omega}=\tan \alpha / 2$. Semiclassically, this value for spin in the $\mathbf{z}$ direction should be $\sigma_{z}=\sin \alpha$, by the same argument given above. Indeed the authors themselves seem to have realized this by the time of their 1988 article ${ }^{(5)}$ A semiclassical analysis could never yield an arbitrarily large value for weak spin. Moreover, no further mention is made of a classical interpretation of weak measurements.

Finally, what is the validity of their claim that $\sigma_{w}$ indeed represents a new quantum variable called the weak variable? We have already seen that 
the interference effect it describes can adequately be derived from the standard variables of quantum mechanics in the ordinary manner. How can the authors propose that they have found a new variable for the measured system when such a value depends on a weakness condition for the measuring device? Perhaps, they are persuaded into assigning reality to this displacement as an actual measuring result because it apparently does not depend explicitly on the measuring coordinate $\pi$. Only the pre- and post-selected state and the observable to be weakly measured factor into the prescription for the weak value. However, we see that a stringent condition of weakness is placed on the measuring device implicitly. It is only due to a relative insignificance of the originally displaced Gaussians after the weak measurement that we have obtained the supposed independence. This is tantamount to saying that $\sin (x)$ are the same function just because of the similarity in their first-order expansion.

We conclude that though it is convenient to label this shift due to interference as a weak quantum number, it is fundamentally misleading, because the measured system does not possess this property in any meaningful way.

\section{ACKNOWLEDGMENTS}

I would like to thank J. Bub for stimulating discussions and suggestions. I would also like to thank C. Y. Ho for her support.

\section{REFERENCES}

1. D. Albert, Y. Aharonov, and S. D'Amato, Phys. Rev. Lett. 54, 5 (1985).

2. J. Bub, and H. Brown, Phys. Rev. Lett. 56, 2337 (1986).

3. L. Vaidman, Y. Aharonov, and D. Albert, Phys. Rev. Lett. 58, 1385 (1987).

4. Y. Aharonov, D. Albert, and L. Vaidman, Phys. Lett. A 124, 199 (1987).

5. Y. Aharonov, D. Albert, and L. Vaidman, Phys. Rev. Lett. 60, 1351 (1988). 